\title{
CCD photometry of YY Orionis stars ${ }^{\star}$
}

\author{
S. Harder ${ }^{1}$, C. Bertout ${ }^{2}$, and C. Mennessier ${ }^{1}$ \\ 1 Laboratoire d'Astrophysique, Observatoire de Grenoble, Université Joseph Fourier, BP. 53, 38041 Grenoble Cedex 9, France \\ ${ }^{2}$ Institut d'Astrophysique de Paris, 98 bis boulevard Arago, 75014 Paris, France
}

Received June 12; accepted September 29, 1997

\begin{abstract}
Photometric $U B V R I$ observations of the YY Orionis stars YY Ori, SY Ori, CE Ori, NS Ori and XX Ori are presented. The luminosity of all stars is variable on a timescale of a few days. This variation is rather irregular and no periodic variability was observed in the present data sets. The $V-I$ and $V-R$ colors become redder when the stars become fainter, suggesting that inhomogeneous surface temperature distributions are responsible for the observed irregular variability. Two stars located in the vicinity of our program stars and observed in the same frames turned out to be variable. They were identified as V481 Ori and AO Ori. We report a periodic modulation of the V481 Ori light curve with a period of 4.9 days.
\end{abstract}

Key words: stars: pre-main sequence - stars: activity — stars: variables: other

\section{Introduction}

The term "YY Orionis star" was introduced by Walker (1972) to define a subclass of classical TTauri stars (CTTSs). Whether this subclass really differs from the CTTS class is still an open question. A bona fide YY Orionis star displays inverse P Cygni absorption components at the edge of the Balmer and Ca II emission lines. These absorption components are red-displaced by typically $300 \mathrm{~km} \mathrm{~s}^{-1}$, which is a direct indication that matter accretes onto the star at close to free-fall velocity.

According to Walker's observations, the YY Orionis phenomenon seems restricted to CTTSs which have a strong UV excess in their spectral energy distribution (Walker 1983). Appenzeller (1977) then found that about $75 \%$ of the YY Orionis stars show a sizable UV excess while the same is true for only around $50 \%$ of all

Send offprint requests to: S. Harder

* Tables 3 to 7 are only available in electronic form at the CDS via anonymous ftp to cdsarc.u-strasbg.fr (130.79.128.5) or via http://cdsweb.u-strasbg.fr/Abstract.html
CTTSs. More recently, high-resolution spectroscopy of a large CTTS sample revealed that inverse P Cygni profiles are present in most CTTSs in at least one of their Balmer lines, though they appear much shallower than in YY Orionis stars (Edwards et al. 1994). Thus the phenomenon of inverse P Cygni profiles seems to be rather ubiquitous in T Tauri stars, and one may doubt that YY Orionis characteristics are defining a peculiar class of young stellar objects. Nevertheless the question of why YY Orionis red-displaced absorption lines are much deeper than in CTTSs still remains.

Since the presence of inverse P Cygni absorption components are a direct indication of accretion of matter onto the star, elucidating the difference between YY Orionis stars and "normal" CTTSs may shed light upon the accretion process itself. Photometric observations in 1994 of class prototype YY Ori (Bertout et al. 1996) revealed a quasi-periodic modulation of its light curve. It was also shown that this modulation was due to the presence of a large hot spot (or group of spots) on the stellar surface. The derived spot area covered about $10 \%$ of the stellar disk area, which is much larger than hot spots usually found in CTTSs. Comparing YY Ori's characteristics to those of CTTSs shows that YY Ori has a much more important accretion rate than usually determined for CTTSs (Hartigan et al. 1995), which suggested a link between the accretion spot size and the accretion rate. However, a comparaison of the spot's luminosities and accretion luminosities of a small sample of CTTSs (including YY Ori) indicated that the spot's luminosity derived from the photometric variability is much smaller than the accretion luminosity of these stars. This and the fact that CTTSs with cold spots have similar accretion rates as CTTSs with hot spots led us to suggest that most of the accretion power might be dissipated in axially or spherically symmetric regions.

We recently observed a sample of YY Orionis stars (taken from Walkers 1972 original list) to study their photometric behaviour and compare them to "normal" CTTSs. Section 2 describes the observations. In Sect. 3 
we present and discuss the results, and we conclude with Sect. 4.

\section{Observations}

We performed $U B V R I^{1}$ differential photometry on the stars YY Ori, SY Ori, CE Ori, NS Ori and XX Ori ${ }^{2}$. The observations took place at the $1 \mathrm{~m}$ telescope of the Wise Observatory in Mizpe Ramon (Israël) from 1996 November 23 to December 2 and from December 23 to 1997 January 2. Additional photometric observations in the $R$ and $V$ band were obtained by $\mathrm{S}$. Allain and $\mathrm{H}$. Geoffray for the star YY Ori at the $120 \mathrm{~cm}$ telescope of the Observatoire de Haute-Provence (France) in the period between December 2 and December 15. A $1024 \times$ 1024 pixel CCD detector was used in both cases to image a field of view of about $12 \times 12$ arcmin. The magnitude of the target star was compared to the average magnitude of a sample of comparison stars on the same CCD frame. Comparison stars were chosen as described in a previous paper (Bertout et al. 1996) to which we refer the reader for additional detail on the data reduction procedure.

\section{Results}

Photometric results are given in Tables 3-7. For the stars NS Ori and XX Ori the quality of the $U$ band measurements was poor and corresponding measurements were thus omitted in the tables. Table 2 shows the ranges of variation observed for each star in each filter. Because of the limited number of observation points, especially in the $B$ and $U$ band, these ranges are likely to be only lower limits of the amplitudes of variation. In the field of YY Ori and CE Ori/AO Ori two reference stars were present. One of them, NGC 1977-38 has known magnitude and spectral type and allowed us to translate the relative magnitudes of YY Ori to absolute magnitudes. The reference star $U B V$ M51765 is of unknown spectral type, but photometric measurements in the $U B V$ bands were made and are used to estimate the $U B V$ magnitudes of CE Ori and AO Ori. The star Parenago 1943 who has known spectral type and $B V$ magnitudes was used to estimate NS Ori's magnitudes. Resulting observed magnitudes are given in Table 1. We applied the periodogram analysis (see Horne \& Baliunas 1986) to the data in order to search for a periodic component. Periods were searched for within the time interval 2-10 days, except for CE Ori for which a interval of 2 to 20 days was taken. We now briefly comment on individual objects.

$1 \quad U, B, V$ and $I$ on Johnson's and $R$ on Cousin's system.

2 Two stars located in the CCD frames of program stars SY Ori and CEOri were found to be variable and are thus discussed here as well. They are V481 Ori and AO Ori.
Table 1. Observed apparent magnitudes

\begin{tabular}{ccccc}
\hline \hline Filter & YY Ori & CE Ori & AO Ori & NS Ori \\
\hline$I$ & 12.09 & - & - & 12.41 \\
$R$ & 12.90 & - & - & 14.01 \\
$V$ & 13.82 & 15.29 & 15.42 & 15.27 \\
$B$ & 14.74 & 15.98 & 16.37 & 16.81 \\
$U$ & 14.69 & 16.00 & 16.73 & - \\
\hline \hline
\end{tabular}

Table 2. Ranges of variation

\begin{tabular}{|c|c|c|c|c|c|c|c|}
\hline Filter & YY Ori & SY Ori & CE Ori & NS Ori & XX Ori & V481 Ori & AO Ori \\
\hline$I$ & 1.14 & 0.30 & 0.55 & 0.29 & $0.62(1.31)$ & 0.22 & 1.84 \\
\hline$R$ & 1.43 & 0.38 & 1.12 & 0.31 & 0.85 & 0.29 & 2.67 \\
\hline V & 2.16 & 0.57 & 1.46 & 0.73 & 1.14 & 0.32 & 3.04 \\
\hline B & 2.62 & 0.62 & 1.89 & $(0.86)$ & $(0.27)$ & 0.46 & 3.60 \\
\hline$U$ & 3.25 & 0.52 & 1.83 & - & - & $(0.40)$ & 4.14 \\
\hline
\end{tabular}

\subsection{YYOri}

YY Ori is an extremely active CTTS with spectral type K5e and a strong $U V$ excess. It is the prototype of this peculiar class of CTTSs. In a previous publication we reported a quasi-periodic modulation of the star's light curve with a period of about 7.6 days and showed that this variation was due to the presence of a hot spot with temperature $6060 \mathrm{~K}$ covering about $10 \%$ of the projected stellar disk area. The present set of observations does not provide any evidence for periodic or quasi-periodic behavior. The light curve shows an irregular variability on the time scale of 3 to 4 days.

\subsection{SYOri}

A possible period of 5.7 days for the K3-star SY Ori was reported by Gahm et al. (1993). They interpreted the variation as being due to the presence of a cold spot on the stellar surface. Applying the periodogram analysis to our data the largest peak for the $I$ band is found at 8.2 days, but with a confidence level of less than $90 \%$. The periodograms of the other filters have no significant peak with a confidence level much more then $50 \%$. The light curve of the first half of our observation campaign shows two minima of different value, while the second half shows more irregular variations with a different shape. The amplitude of variation is less than $1 \mathrm{mag}$ in all filters although there are not enough measurements in the $U$ band to accuratly determine the amplitude of the $U$ variability. If it is well correlated with the variability in the other filters, one may expect the amplitude in $U$ to exceed 1 mag. The peculiar form of the light curve with two different values of the minima stands in contrast to simple spot models. 


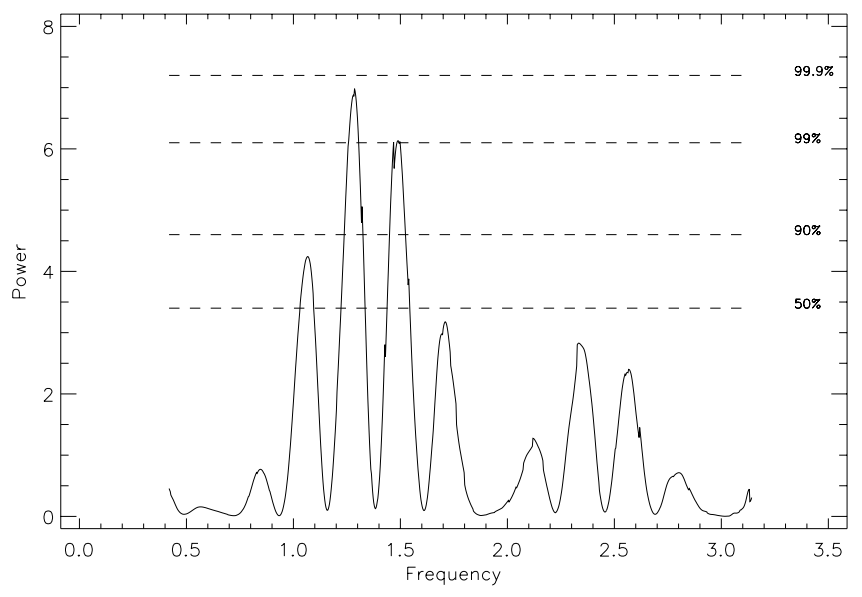

Fig. 1. Periodogram of V481 Ori in filter $I$. The dashed horizontal lines respresent the confidence levels

\section{3. $C E O r i$}

CE Ori is a binary system (Hartigan et al. 1994) where the stars are separated by $13.7^{\prime \prime}$ and have spectral types M1 and M3. Applying the periodogram analysis, one peak is found at $\approx 11$ days in the filters $I, R$ and $V$ with a confidence level at about $99 \%$. Also, a second equally important peak arises between 18 and 19 days. Since each observation intervall was about 10 days, it is impossible to determine a reliable period. The amplitude of variation is more than 1 mag in filter $R$ and $V$ which rules out that a cold spot causes the brightness modulation, since T Tauri stars with cool spots show typical amplitudes of variation of less than 1 mag in filter $V$ (see Herbst et al. 1994). The maximum is quite flat, in contrast to CTTSs with spots which show a more sinusoidal modulation. It is also interesting that the light curve decreases slowly in the first half of our observation campaign while it seems to drop off rather rapidly in the second half.

\subsection{NSOri}

NS Ori was identified as a flare star by Haro (1976) and has a spectral type of M1. The variability is quite irregular and there is only a weak correlation between the variations in different filters. However, the error is on the same order of magnitude as the variations.

\subsection{Ori}

XX Ori is a flare star (Haro 1976) with spectral type M2.5. The magnitude variations of XX Ori in the different filters are quite well correlated and there seems to be a quasi-periodic behaviour in the first half of the light curve. However, the periodogram analysis did not confirm the presence of a well-defined period in the data set.

\subsection{Additional stars}

\subsubsection{V481 Ori}

V481 Ori is of unknown spectral type but is likely to be a $\mathrm{T}$ Tauri star. Duncan (1993) measured a $v \sin i$ of $21 \mathrm{~km} \mathrm{~s}^{-1}$ and found that it is probably not a binary system. Furthermore the star was identified as a X-ray source by Gagné et al. (1995). The result of the periodogram analysis shows a maximum at a period of 4.89 days with a confidence level of at least $99 \%$ in all filters except $U$ where there are too few observations. A second equally important peak arises at period of 4.2 days. Figure 1 displays the periodogram in the filter $I$ and Fig. 3 shows the light curve with a sinusoidal fit of 4.9 day period and Fig. 4 displays the phased sinus curve fitted to the phased data points. The rms error are typically of the order of 0.05 .

\subsubsection{AO Ori}

The spectral type is not known for AO Ori. The light curves show important amplitudes in all filters: from less than 2 mag in the $I$ band to more than 4 mag in the filter $U$. The dramatic changes in photometry may suggest that it is a CTTS. The periodogram shows no significant peak.

\section{Discussion}

Classical T Tauri stars usually display irregular light variability on many time-scales, but sometimes also show a periodic or quasi-periodic modulation of their light curve on a time scale of a few days (Bouvier et al. 1986, 1993; Vrba et al. 1986, 1989). This quasi-periodic variation is linked to the rotation of the star and can be reproduced by models of inhomogeneous surface temperature distribution. In several cases one finds that spots responsible for the light curve variations are hot; they are interpreted as the shock regions of an infalling flow of matter. In other CTTSs, however, the signatures are typical of dark, magnetic spots. It can be shown that hot spots dominate the light curve when they are present on the stellar surface, even in the presence of dark magnetic spots (cf. Bertout et al. 1996)

Irregular variability is more difficult to interpret and various mechanism have been proposed in the literature (Gahm et al. 1993; Worden et al. 1981; Guenther \& Hessman 1993; Herbig \& Soderblom 1980). Because it is likely ubiquitous in CTTSs, accretion is the most probable process causing the irregular variations in CTTSs. Vrba et al. (1985) showed that the colors of stars with periodic and non-periodic variations of their data set could be reproduced by variations of hot plage-like regions on the stellar surface. Furthermore there seems to be a strong correlation between the overall amplitude of light variations and the variation of $\mathrm{H}_{\alpha}$ equilavent width (Vrba et al. 1993). This suggests that both phenomenon are caused by the same mechanism. Further arguments are discussed by 

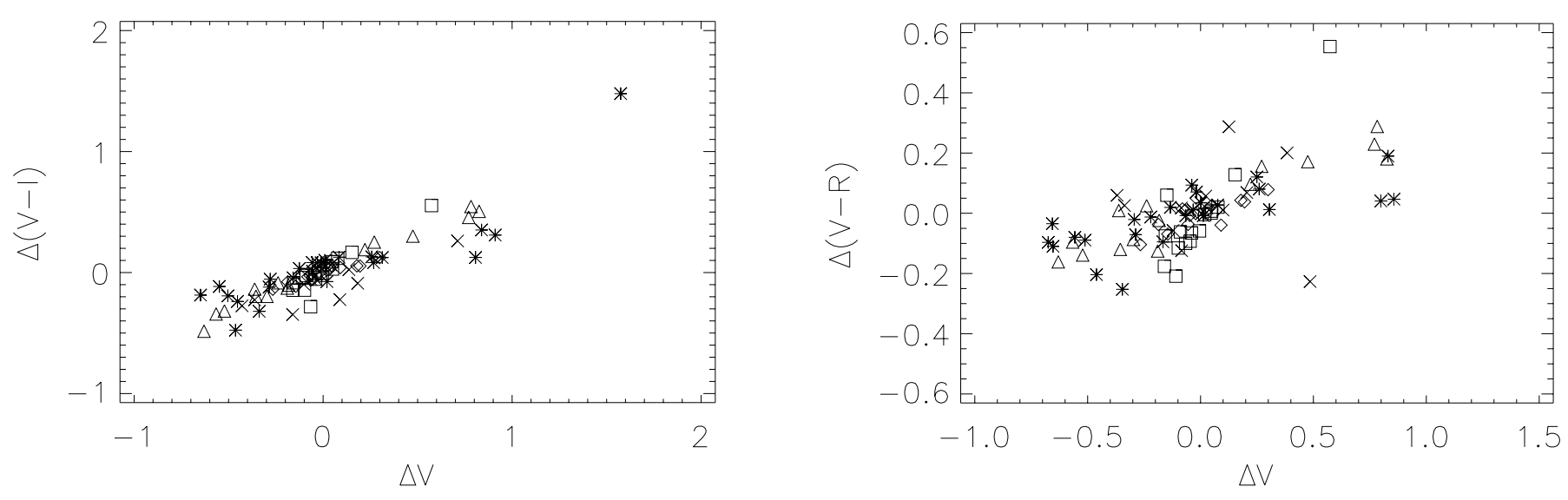

Fig. 2. Plots of $V-I$ and $V-R$ color changes as a function of $V$ magnitude changes for all 5 YY Orionis stars

Herbst et al. (1994) who showed that hot spots responsible for the photometric variability and the hot components producing the veiling are likely to be the same.

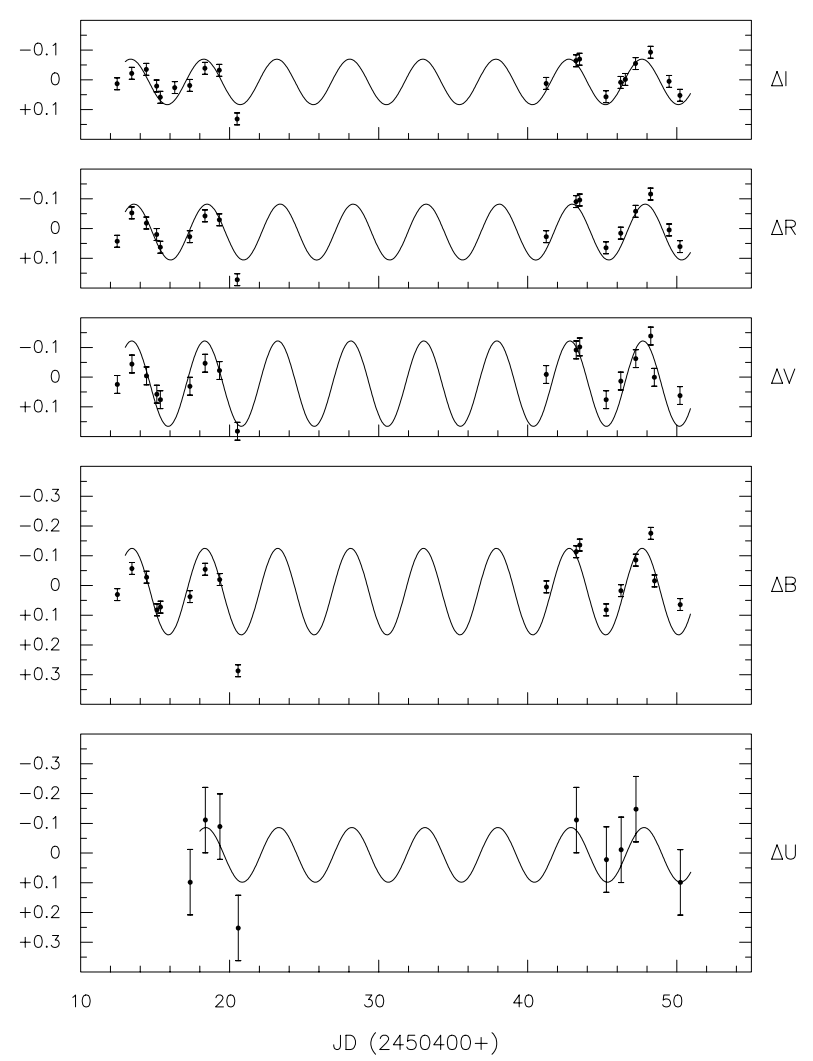

Fig. 3. CCD photometric observations of V481 Ori in $U B V R I$. The magnitude values given here refer to the deviation from the average observed magnitude. The solid lines shows the best-fit sine curves for a period of 4.9 days

As it can be seen in Fig. 2, all stars get redder in the color $V-I$ and $V-R$ when the $V$ magnitude becomes fainter. There is more scatter in $B-V$ but the same trend
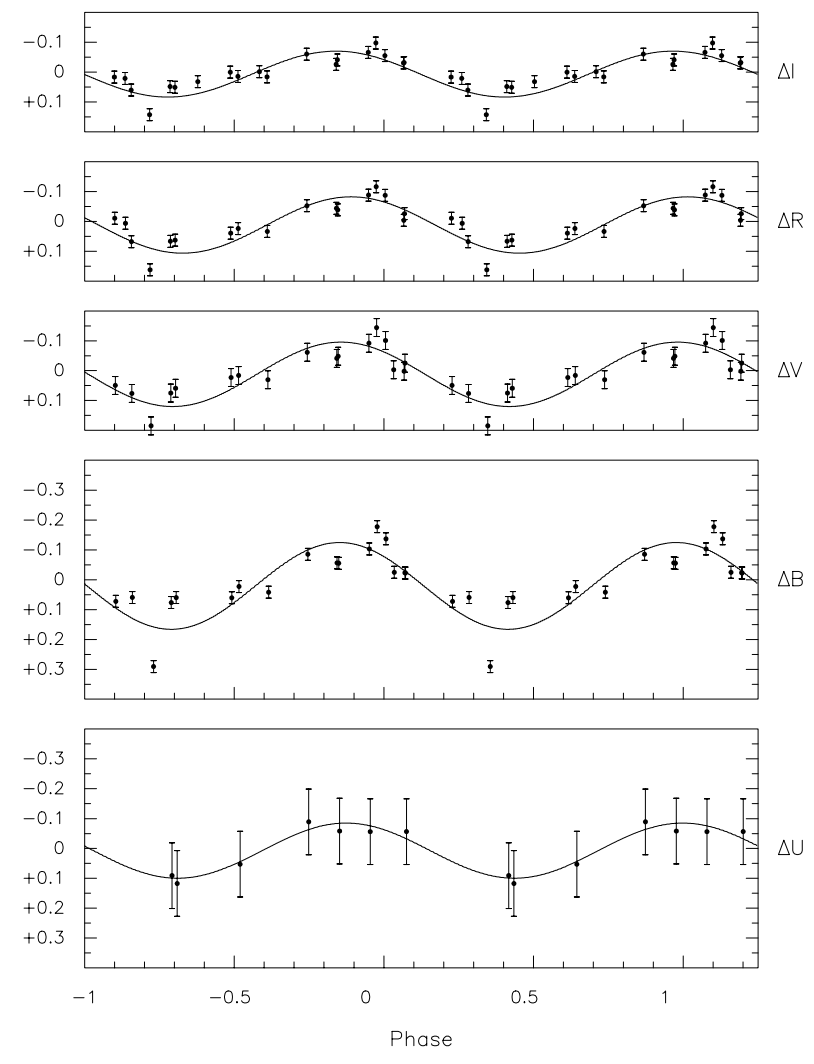

Fig. 4. Phased folded photometric data for V481 Ori. The solid lines represent the best sinusoidal fit to the data in each filter

seems to be present in at least some stars, although one has to caution that the measurement errors is quite large in $B$. The behavior of the $V-I$ and $V-R$ colors of the YY Orionis stars in the observed sample thus resembles that observed in CTTSs, and can be explained by the presence on the stellar surface of an additional variable blackbody-like source with a temperature different from the stellar effective temperature. 
The photometric behavior of our program stars however does not give any further insight in the characteristics of hot spots found on YY Orionis stars, since we did not observe a periodic modulation of their light curves. Like CTTSs, they display most of the time an irregular variability. This means that hot spots may be distributed more or less homogeneously on the stellar surface most of the time. It is only when one group of spots becomes dominant and survives at least one rotation period that a periodic or quasi-periodic variation can be observed.

In order to gain insight into the physical connection between photometric variability and accretion it is necessary to perform spectroscopic observations on our program stars with simultaneous photometry. We shall then be able to study possible correlations of the red-displaced absorption components with photometry. This will be done in a future observation campaign.

Acknowledgements. We are indebted to the director and staff of Wise Observatory for time allocation and logistic help. We would like to thank Stéphanie Allain and Hervé Geoffray for providing us additional observations of YY Ori obtained at the OHP. This research has made use of the Simbad data base, operated at CDS, Strasbourg, France.

\section{References}

Appenzeller I., 1977, in: The Interaction of Variable Stars with their Environment, Kippenhahn R., Rahe J., and Strohmeier W. (eds.). Remeis-Sternwarte, Bamberg, p. 80
Bouvier J., Bertout C., 1986, A\&A 211, 99

Bouvier J., Cabrit S., Fernandez M., Martin E.L., Matthews J.M, 1993, A\&AS 101, 485

Bertout C., Harder S., Malbet F., Mennessier C., Regev O., 1996, AJ 112, 2159

Duncan D.K, 1993, ApJ 406, 172

Edwards S., Hartigan P., Ghandour L., Andrulis C., 1994, AJ 108, 1056

Gagné M., Caillault JP., Stauffer J.R., 1995, ApJ 445, 280

Gahm G.F., Gullbring E., Fischerström C., Lindroos K.P, Lodén, 1993, A\&AS 100, 371

Guenther E., Hessman F.V., 1993, A\&A 268, 192

Haro G., 1976, Bol. Inst. Tonantzintla, 2, 3-54

Hartigan P., Strom K.M, Strom S.E., 1994, ApJ 427, 961

Hartigan P., Edwards S., Ghandour L., 1995, ApJ 452, 736

Herbst W., Herbst D.K, Grossman E.J., Weinstein D., 1994, AJ 108, 1906

Herbig G.H, Soderblom D.R., 1980, ApJ 242, 628

Horne J.H., Baliunas S.L, 1986, ApJ 302, 757

Vrba, F.J., Rydgren A.E., Zak D.S., Schmelz J.T., 1985, AJ 90,326

Vrba, F.J., Rydgren A.E, Chugainov P.F., Shakovskaya N.I, Zak D.S., 1986, 306, 199

Vrba, F.J., Rydgren A.E, Chugainov P.F., Shakovskaya N.I, Weaver W.B, 1989, AJ 97, 483

Vrba, F.J., Chugainov P.F., Weaver W.B, Stauffer J.S., 1993, AJ 106,1608

Walker M.F, 1972, ApJ 175, 8

Walker M.F., 1983, ApJ 271, 642

Worden S.P., Schneeberger T.J., Kuhn J.R., Africano J.L., 1981, ApJ 244, 520 